\title{
Accumulation of myeloid lineage cells is mapping out liver fibrosis post injury: a targetable lesion using Ketanserin
}

\author{
Saeid Amini-Nik $\mathbb{D}^{1,2,3}$, Ali-Reza Sadri ${ }^{1,4}$, Li Diao ${ }^{1,4}$, Cassandra Belo ${ }^{1}$ and Marc G. Jeschke 1,3,4,5,6
}

\begin{abstract}
Liver fibrosis is problematic after persistent injury. However, little is known about its response to an acute insult. Accumulation of myeloid lineage cells contributes into the promotion and resolution of inflammation and fibrosis. Using Cre-transgenic mice that specifically mark myeloid lineage cells with EYFP and burn as a model of acute systemic injury, we investigated the role of myeloid lineage cells in the liver after acute injury. Our data show that thermal injury in mice (30\% total body surface area) induces fibrosis predominantly around portal venules whereas myeloid cells are enriched throughout the liver. The fibrosis peaks around 1-2 weeks post injury and resolves by week 3. Ablating myeloid cells led to lower fibrosis. Through FACS sorting, we isolated myeloid lineage cells (EYFP + ve cells) from injured animals and from the control uninjured animals and subjected the extracted RNA from these cells to microarray analysis. Microarray analysis revealed an inflammatory signature for EYFP + ve cells isolated from injured animals in comparison with control cells. Moreover, it showed modulation of components of the serotonin (5-HT) pathway in myeloid cells. Antagonizing the $5 \mathrm{HT}_{2 \mathrm{~A} / 2 \mathrm{C}}$ receptor decreased fibrosis in thermally injured mice by skewing macrophages away from their pro-fibrotic phenotype. Macrophages conditioned with Ketanserin showed a lower profibrotic phenotype in a co-culture system with mesenchymal cells. There is a spatiotemporal pattern in liver fibrosis post-thermal injury, which is associated with the influx of myeloid cells. Treating mice with a $5 \mathrm{HT}_{2 \mathrm{~A} / 2 \mathrm{C}}$ receptor antagonist promotes an anti-fibrotic effect, through modulating the phenotype of macrophages.
\end{abstract}

\section{Introduction}

Severe burn injury results in a systemic response with substantial hepatic alterations ${ }^{1}$. The liver has a diverse role in response to a thermal injury, such as producing acute phase proteins and regulating the systemic inflammatory response ${ }^{1}$. Although several reports show there is a hypermetabolic response, increased inflammation, and endoplasmic reticulum (ER) stress in the liver postthermal injury ${ }^{2,3}$, the spatial pattern and nature of this damage are still a mystery.

\footnotetext{
Correspondence: Saeid Amini-Nik (saeid.amininik@utoronto.ca) or

Marc G. Jeschke (marc.jeschke@sunnybrook.ca)

${ }^{1}$ Sunnybrook Research Institute, Toronto, Canada

${ }^{2}$ Laboratory Medicine and Pathobiology, University of Toronto, Toronto,

Canada

Full list of author information is available at the end of the article.

These authors contributed equally: Saeid Amini-Nik, Ali-Reza Sadri
}

Upon liver injury, there is a wound-healing response that involves extracellular matrix (ECM) deposition ${ }^{4}$. This is a vital reaction for the liver to protect or repair itself ${ }^{5}$. It has been shown that after an acute injury there is fibrosis, which works to shield hepatocytes from toxins rather than having any detrimental role ${ }^{6}$. However, clinically fibrosis is an undesirable event and seen as a form of pathology.

For a long time macrophages have been known to be the promoters of fibrosis but more recently have been shown to be essential for fibrosis resolution as well ${ }^{7-9}$. Macrophages have numerous functional states, which in vitro are normally labeled as M1 (classically activated) macrophages or M2 (alternatively activated) macrophages. M1 macrophages have pro-inflammatory functions whereas M2 macrophages promote resolution of inflammation and wound healing ${ }^{10,11}$. Though, the classification of 
macrophages in vivo is not as black and white. Macrophage heterogeneity is highly complex, as these cells can switch between phenotypes depending on the environmental cues, making it difficult to fully characterize these cells in vivo. Thus, it is best to classify these cells based on functionality rather than marker-based phenotype. Due to their programming versatility and essential role in all stages of wound healing, macrophages have become a therapeutic target for inflammatory and fibrotic conditions $^{12,13}$.

One potential therapeutic remedy for liver injury that is currently being investigated in the field is serotonin (5hydroxytryptamine, 5HT). Primarily known as a neurotransmitter, 5HT, has an essential role in mood, cognition, feeding, and sleep ${ }^{14}$. However, $90 \%$ of 5 -HT is produced outside the CNS, mainly found in the gut ${ }^{15}$. The role of 5 HT includes cell proliferation, vascular contraction and relaxation, apoptosis, and platelet aggregation. Current studies on rodents and humans suggest that 5-HT has an imperative role in liver regeneration and fibrosis ${ }^{16-19}$.

Here, using thermal injury as a model of systemic injury and Cre-transgenic myeloid reporter mice, we show that there is a spatiotemporal pattern in liver fibrosis postthermal injury and myeloid lineage cells orchestrate this fibrotic response. Treating mice with Ketanserin decreases portal fibrosis possibly by skewing the phenotype of myeloid cells away from their pro-fibrotic form. To the best of our knowledge, this is the first study to look at fibrosis in the liver post-thermal injury and show a spatiotemporal pattern of fibrosis after severe trauma.

\section{Materials and methods Mice}

We used our previously reported myeloid lineage reporter mice ${ }^{7}$. Briefly, generation of myeloid lineage reporter mice was done using the Cre-loxP system. To drive Cre in Lysz positive cells only, we used the Lysz-Cre (B6.129-Lysztm1(cre)Ifo/J) mice. Our reporter gene is derived from ROSA-EYFP (B6.129 $\times 1$-Gt[ROSA] 26Sortm1(EYFP)Cos/J) mice, which contain an EYFP gene inserted downstream of a floxed stop codon. Breeding these mice with mice expressing recombinase results in excision of the floxed stop codon and expression of EYFP. The progeny of these mice are called Lysz-Cre; ROSA-EYFP in this manuscript.

\section{Experimental protocol (burn)}

Animal procedures were reviewed and approved by Sunnybrook Research Institute and Sunnybrook Health Sciences Centre at University of Toronto animal care and use committee. Mice were subjected to a full-thickness scald burn. Briefly, animals were anesthetized with inhaled isofluorane and received an intraperitoneal (IP) injection of the analgesic buprenorphine. The dorsum of the animal was shaved and lactated ringer's solution was IP injected along the spine. A 30\% total body surface area (TBSA) thermal injury was induced by placing the animal on a mold that exposes the dorsum ${ }^{20}$. The dorsum of the animal is then exposed to water pre-heated to $98^{\circ} \mathrm{C}$. The exposure lasts for $10 \mathrm{~s}$. The animals are placed in separate cages post-thermal injury. Sham animals received the prep but did not receive a thermal injury.

\section{Histological examination}

Masson's Trichrome and immunohistochemistry was done as described before ${ }^{21,22}$. For immunofluorescence staining, liver sections were blocked ( $1 \%$ bovine serum albumin in $0.5 \%$ PBS-TritonX) and incubated in primary antibodies: F4/80 (AbD Serotec), GFP (Rockland), and BrdU (Cell Signaling). The primary antibodies were detected using AF488 (Vector) and AF647 (Vector). Proliferation in hepatic cells was measured using BrdU uptake. Mice received an IP injection of BrdU $24 \mathrm{~h}$ prior to death $(50 \mathrm{mg} / \mathrm{kg}$ of animal weight).

\section{Image acquisition and image analysis}

Image capture of liver sections stained via immunofluorescence was done using the Zeiss observer Z1 spinning disc confocal microscope. Sections stained via immunohistochemistry were imaged using the Zeiss Axiovert 200 light microscope at $20 \times$ and $40 \times$ magnification for quantification.

\section{Fibrosis index}

Photoshop CS5 was used to measure collagen in the portal zone. We used the color range option to measure blue pixels in the uploaded tiff file image of liver sections stained using Masson's Trichrome protocol. We then did a pixel count of the area of the portal venule, which was used to get a ratio (pixel count of collagen/pixel count of portal venule area) to control for vessel size. The ratio was multiplied by 1000 giving us the fibrosis index.

\section{Western blotting}

Antibody against Desmin (PA5-16705) was purchased from Thermo Fisher Scientific (Rockford, IL, USA); F4/80 (ab186073) was purchased from Abcam (Cambridge, MA, USA); and GAPDH (14C10, \#2118) was purchased from Cell Signaling (Danvers, MA, USA). Clarity Western ECL substrate was purchased from Bio-Rad (Hercules, CA, USA). Liver homogenates ( $50 \mu \mathrm{g}$ of protein per well) were separated by $10 \%$ SDS-PAGE gel, proteins were transferred to nitrocellulose membrane, and then blots were probed using the antibodies listed above. Band intensities were detected, normalized and quantified with the Chemidoc and Image Lab 5.0 software (Bio-Rad Laboratories, Hercules, CA). GAPDH was used as loading control. 


\section{Clodronate}

Liposomal clodronate was purchased from Dr. Nico van Rooijen (www.clodronateliposomes.org). They were prepared as previously described van ${ }^{23}$. Wild-type C57/BL6 mice were purchased from Jackson laboratories. These mice received an IP injection of liposomal clodronate $(0.1$ $\mathrm{mL} / 10 \mathrm{~g}$ of animal tissue) or PBS $48 \mathrm{~h}$ prior to a thermal injury and $48 \mathrm{~h}$ post-thermal injury.

\section{Flow cytometry and microarray analysis}

Mice with the same genotype (Lysz-Cre;ROSA-EYFP) were exposed to a $30 \%$ thermal injury or had sham preparation. They were monitored for 1 week prior to harvesting. The liver was removed from each mouse and digested with collagenase cocktail $(1000 \mathrm{U} / \mathrm{ml}$ collagenase, $1 \mathrm{X}$ dispase, $0.05 \%$ trypsin-EDTA in DMEM with $1 \% \mathrm{Ab} /$ Am) for $30 \mathrm{~min}$ at $37^{\circ} \mathrm{C}$. For isolation of EYFP+ cells, half of the dissociated cells were taken from each mouse, mixed and subjected to BD FACS ARIA II for flow cytometry analysis and sorting of the cells. The EYFP+ population was sorted and sham provided 600,000 cells and burn provided 1,300,000 cells. These cells were stored at $-80^{\circ} \mathrm{C}$ until RNA preparation. The remaining half of the liver were sampled through flow cytometry separately and probing for EYFP, CD11b (eBioscience, APC-eFluor 780) and F4/80 (Biolegend, PerCP cy5.5). The flow cytometer used for cell analysis was a Cytek DxP FACSCalibur. For RNA Preparation TRIzol reagent (Life Technologies) was used to lyse the cells. The manufacturer's instructions were followed. In short, $1 \mathrm{ml}$ of TRIzol was added to each cell pellet. Chloroform was added and the samples were centrifuged at 12,000 $g$ for 15 $\min$ at $4{ }^{\circ} \mathrm{C}$. The aqueous phase was carefully removed and transferred to a new tube. RNA was precipitated with $100 \%$ isopropanol and centrifuged at $12,000 \mathrm{~g}$ for $10 \mathrm{~min}$ at $4{ }^{\circ} \mathrm{C}$. The supernatant was removed and the RNA pellet was washed with $75 \%$ ethanol, centrifuged at $7500 \mathrm{~g}$ for 5 $\min$ at $4{ }^{\circ} \mathrm{C}$. The pellet was air dried and resuspended in RNase-free water. The absorbance at 260 and $280 \mathrm{~nm}$ was measured to determine the RNA yield. For cDNA synthesis, $2 \mu$ g of RNA was taken and used with the kit HighCapacity cDNA Reverse Transcription Kit (Life Technologies) according to the manufacturer's instructions.

\section{Ketanserin treatment}

Wild-type C57BL/6 mice were treated with $10 \mathrm{mg} / \mathrm{kg}$ of Ketanserin daily for 7 days prior to the thermal injury and treated for another 14 days post-thermal injury. For in vitro experiments, Ketanserin was diluted in sterile saline. Bone-marrow-derived macrophages (BMDMs) were cultured for 7 days. On day 6 and 7 macrophages were treated with $10^{-9} \mathrm{M}$ of Ketanserin.

\section{Cell culture}

BMDMs were retrieved from wild-type C57BL/6 mice. Cells were cultured in macrophage medium consisting of RPMI-1640, 10\% fetal bovine serum, 10\% L929, and 1\% antibiotics-antimicrobial solution. Cells were cultured at $37^{\circ} \mathrm{C}$ in a humidified atmosphere with $5 \%$ carbon dioxide for 7 days.

\section{Co-culture of macrophages and BMD-MSCs}

FalconTM cell culture inserts for 6 well plates were used to co-culture BMDMs with BMDMSCs. Macrophages and MSCs were first cultured in separate 6-well plates for 7 days to allow maturation of cells. Macrophages were cultured in macrophage media, which is described above. MSCs were cultured in MSC conditioned media consisting of MesenCult ${ }^{\mathrm{m}}$ MSC Basal Medium (Mouse), Mesenchymal Stem Cell Stimulatory Supplements (Mouse) and MesenPure ${ }^{\mathrm{ma}}$. Macrophages are added to the wells and inserts. On days 6 and 7 macrophages are treated with Ketanserin or saline and on day 10 inserts with macrophages pretreated with Ketanserin or saline are added to six well plates with MSCs at the bottom. The two cell types are co-cultured for 3 days.

\section{Phagocytosis assay}

Fluorescently labeled zymosan particles (Life technologies) were centrifuged onto the BMDMs at $450 \mathrm{~g}$ for 2 min. The samples were then incubated at $37^{\circ} \mathrm{C}, 5 \% \mathrm{CO}_{2}$ for $1 \mathrm{~h}$. Phagocytosis was stopped by ice-cold PBS followed by four washes and cells fixed in 4\% PFA for 20 min. This was followed by two more washes with PBS. Cells were permeabilized with PBS containing 1\% BSA and $0.5 \%$ Triton X-100 for $15 \mathrm{~min}$. Cells were washed three more times with PBS containing $1 \%$ BSA. Cells were then stained with FITC-labeled phalloidin and Dapi.

\section{RNA isolation and real-time quantitative polymerase chain reaction}

TRIzol reagent (Invitrogen, Carlsbad, CA, USA) was used to isolate RNA according to manufacturer's instructions. The NanoDrop-2000 spectrophotometer (ThermoScientific, Waltham, MA, USA) was used to determine total RNA yield. cDNA synthesis was done in a thermocycler (AB Applied Biosystems, Foster City, CA, USA), after mixing $1 \mu \mathrm{g}$ RNA and a master mix prepared with the high-capacity CDNA synthesis reverse transcription kit (AB Applied Biosystems). SYBR ${ }^{\circledast}$ Green PCR Master Mix (Applied Biosystems) was used to perform a Quantitative polymerase chain reaction (PCR) looking at the following genes of interest: $I l-6, I l-10, T g f-\beta 1, T g f-\beta 3$, Lcn-2, iNos, Arg, and Tnf- $\alpha$. 


\section{Analysis of liver function}

Prior to sacrificing of mice, $0.5 \mathrm{~mL}$ of blood was collected and serum was separated by centrifugation at 3000 rpm for $20 \mathrm{~min}$. Serum AST and ALT levels were measured using AST and ALT Activity Assay Kits (SigmaAldrich).

\section{Statistical analysis}

Statistical comparisons between the groups were performed using an unpaired student's $t$-test. A two-tailed $p$ value $\leq 0.05$ was considered significant. Data were graphically expressed as the mean of the target group \pm the standard error of the mean.

\section{Results}

\section{Thermal injury results in liver fibrosis with a unique spatiotemporal pattern}

Knowing that there is a systemic inflammatory response and profound liver dysfunction post-thermal injury; we investigated whether thermal injury induces a fibrotic response in the liver. A mild temporal pattern in liver fibrosis was observed (Fig. 1a). After a 30\% TBSA fullthickness burn, mice were sacrificed at 2, 7, 14, 21, and 42 days post-thermal injury. The comparison was made between age-matched uninjured litter-mates (control sham group). Liver fibrosis was assessed using Masson's Trichrome staining. Hepatic fibrosis peaked between 7 and 14 days post-thermal injury, while its resolution occurred by day 21 and remained low at day 42 (Fig. 1a). The observed fibrosis was comparable to the F1 stage of the metavir score, portal fibrosis without septae ${ }^{24}$. Interestingly, the observed fibrosis is found only in the portal field, predominantly around portal venules (PV) rather than around the central veins $(\mathrm{CV})$ (Fig. 1b). In addition, we observed a decrease in quiescent hepatic stellate cells (desmin + cells), the resident mesenchymal stem cells of the liver, after thermal injury (Fig. 1c, d). This suggests that they have transitioned into an activated state as myofibroblasts, the key producers of collagen.

\section{Myeloid lineage cells accumulate in the liver post-thermal injury}

Having identified the time when there is maximal fibrosis, at roughly 7 days post-thermal injury, we investigated whether there is an accumulation of myeloid cells in the liver at that time point. Through flow cytometry, we observed a significant increase in EYFP + cells and cd11b + cells (Fig. 2a). The percentage of EYFP + cells that are cd11b+ increases post-thermal injury suggesting that these cells are from an extra-hepatic source (Supporting Fig.1A). Double staining of EYFP and F4/80 in the liver shows that $90 \%$ of EYFP + cells are also F4/80+ (Supporting Fig.1B, C). We also observed an increase in the percentage of EYFP + cells in the bone marrow postthermal injury, suggesting that systemic thermal injury mobilizes bone marrow cells and shift bone marrow fate more toward a myeloid lineage (Supporting Fig. 2A and 2B). Immunohistochemical staining showed a significant increase in myeloid cells (Fig. 2b) and F4/80 positive macrophages at 7 days' post-thermal injury (Fig. 2c). Interestingly, accumulation of EYFP + cells and F4/80+ cells were observed in the portal field. To find out if the increase in myeloid lineage cells in the liver is due to recruitment or proliferation of EYFP+ cells, we did double staining for EYFP and BrdU. We observed a very limited number of EYFP+ cells that were BrdU+, comparable with sham mice, at 1 and 2 weeks post-thermal injury, suggesting that accumulation of myeloid cells is mainly due to recruitment (Supporting Fig. 1D).

\section{Ablating macrophages prevents fibrosis in the liver post- thermal injury}

To verify whether accumulation of myeloid lineage cells and its spatial presence in the portal field is essential for fibrosis, we treated mice with $0.1 \mathrm{~mL} / 10 \mathrm{~g}$ of liposomal clodronate through IP injection pre and post-thermal injury. We observed less fibrosis at day 7 post-thermal injury in clodronate-treated mice in comparison to PBS treated burned mice (Fig. 3a). Ablation of macrophages was not limited to the portal field. We observed a global ablation of macrophages in the liver (Fig. 3b-d). Furthermore, when macrophages are ablated pre- and postthermal injury, we do not observe a reduction in desmin + cells in the liver compared to when macrophages are present (Fig. 3d). This suggests that macrophages are essential in activating hepatic stellate cells into their profibrotic form.

\section{Liver myeloid lineage cells show a gene expression profile favoring inflammation and fibrosis}

We showed that myeloid cells are critical for liver fibrosis post-thermal injury. Then, we wanted to identify genes or signaling pathways that are modulated in myeloid cells after a severe burn and identify underlying mechanisms in myeloid cells that promote fibrosis in the liver. Myeloid lineage cells tagged with EYFP were sorted via FACS from sham-treated and burned mice 7 days after injury. Gene expression analysis (Affymetrix mouse gene 2.0 st array) was performed.

Considering only genes with a fold change $>2$ in the analysis and comparing burn with sham samples, we observed an upregulation of 856 genes and downregulation of 1170 genes (Fig. 4a). The microarray data suggest at 7 days post-thermal injury there is upregulation of numerous genes associated with wound healing and restorative macrophages, although the majority of upregulated genes highlight a pro-inflammatory phenotype in 

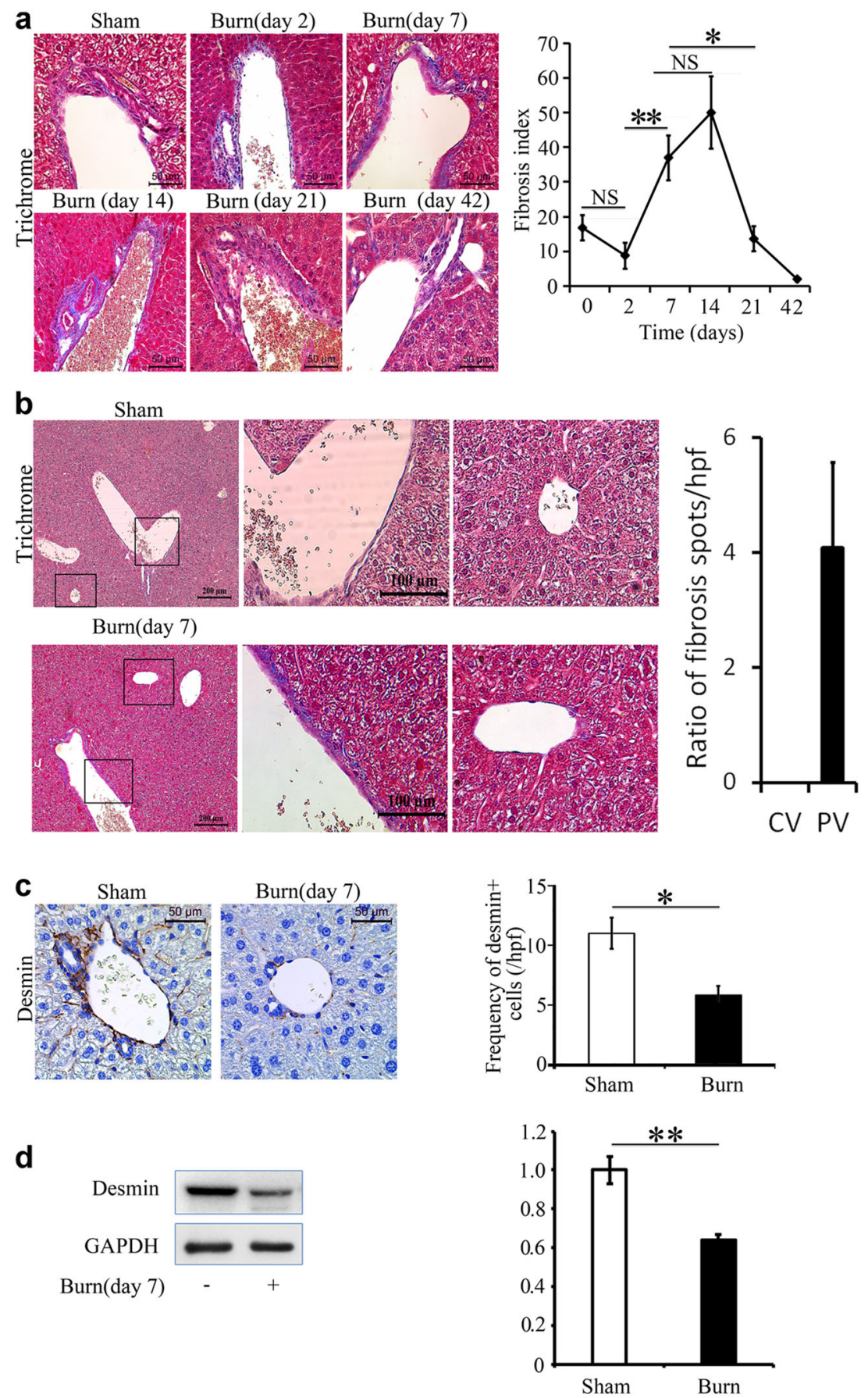

Fig. 1 Thermal injury primes liver fibrosis in a spatiotemporal pattern. a There is a temporal trend in fibrosis around PVs post-thermal injury. Mice were examined after $2,7,14,21$, and 42 days post-thermal injury; $n=5-7$, scale bar $50 \mu \mathrm{m},{ }^{*} P<0.05,{ }^{* *} P<0.001$. NS not significant. b Trichrome staining shows that collagen is deposited predominately around PVs rather than CVs. c-d Thermal injury promotes a reduction in desmin + cells $n=$ 3-5

these cells (Fig. 4b). Comparison of expression arrays (Gene Expression Omnibus database, accession no.,...) using ingenuity pathway analysis showed myeloid lineage cells were involved in numerous pathways associated with inflammation, fibrogenesis and the coagulation cascade (Supporting Fig. 3A, B). More specifically, stellate cell activation (Supporting Fig. 4A), and 5HT pathways (Fig. 4c) are some of the deregulated pathways in myeloid cells of injured animals in comparison with the sham control group.

In addition, we analyzed genes associated with inflammation and wound healing to so see if myeloid cells in the 

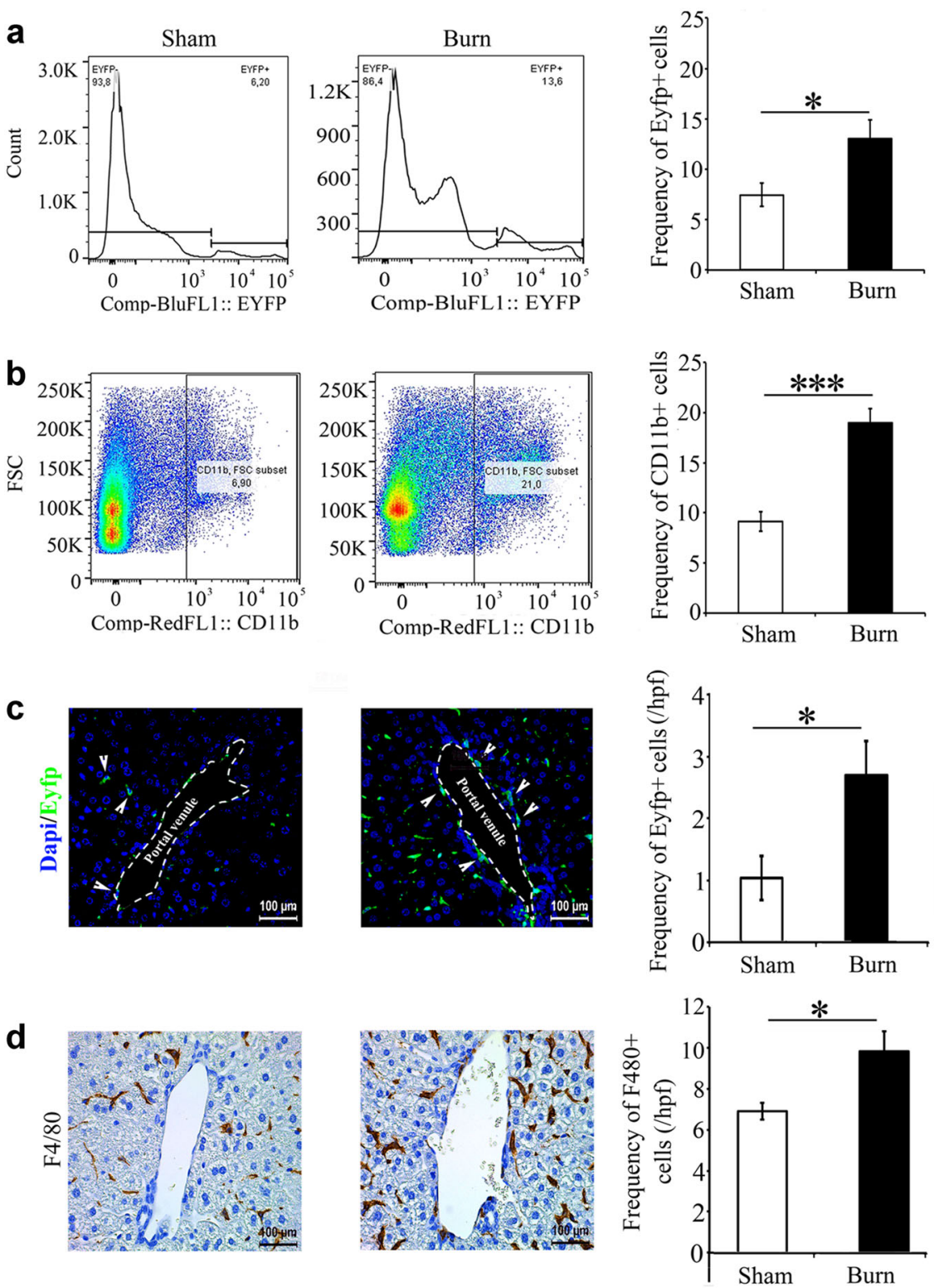

Fig. 2 Myeloid cells are recruited to the liver post-thermal injury. $\mathbf{a}$, b Flow cytometry shows an increase in EYFP + and $c d 11 b+c e l l s . \mathbf{c}$ Immunofluorescence of Lysz-Cre ROSA-EYFP livers shows an enrichment of EYFP+ cells post-thermal injury, in particular around PVs. scale bar 100 $\mu \mathrm{m}$. d Immunohistochemistry staining for $\mathrm{F} 4 / 80+$ cells show an increase post-thermal injury in these cells in the liver and surrounding PV sites; $n=5$ for all the panels, scale bar $100 \mu \mathrm{m} .{ }^{*} P<0.05$, and ${ }^{* *} P<0.001$

liver are polarized towards a particular phenotype 7 days post-thermal injury.

\section{Treating mice with the $5 \mathrm{HT}_{2 \mathrm{~A} / 2 \mathrm{C}}$ antagonist Ketanserin results in less liver fibrosis}

5 HT has been suggested to be a key molecule in hepatic regeneration. Our pathway analysis revealed that several components of $5 \mathrm{HT}$ pathway have been modulated in the myeloid lineage cells post-thermal injury (Fig. 4c). Thus, we investigated its effects on liver fibrosis post-thermal injury. We observed less fibrosis in mice treated with Ketanserin (Fig. 5a) with no additional detrimental effect on the liver function according to our AST/ALT test on blood derived from thermally injured mice, mice that received Ketanserin post-thermal injury and uninjured sham control mice (Supporting Fig. 4B). To verify whether the anti-fibrotic effect of Ketanserin is due to a change in the spatial accumulation of myeloid cells in the portal field or due to change in the inflammatory profile of these cells, we quantified the number of F4/80+ cells in 

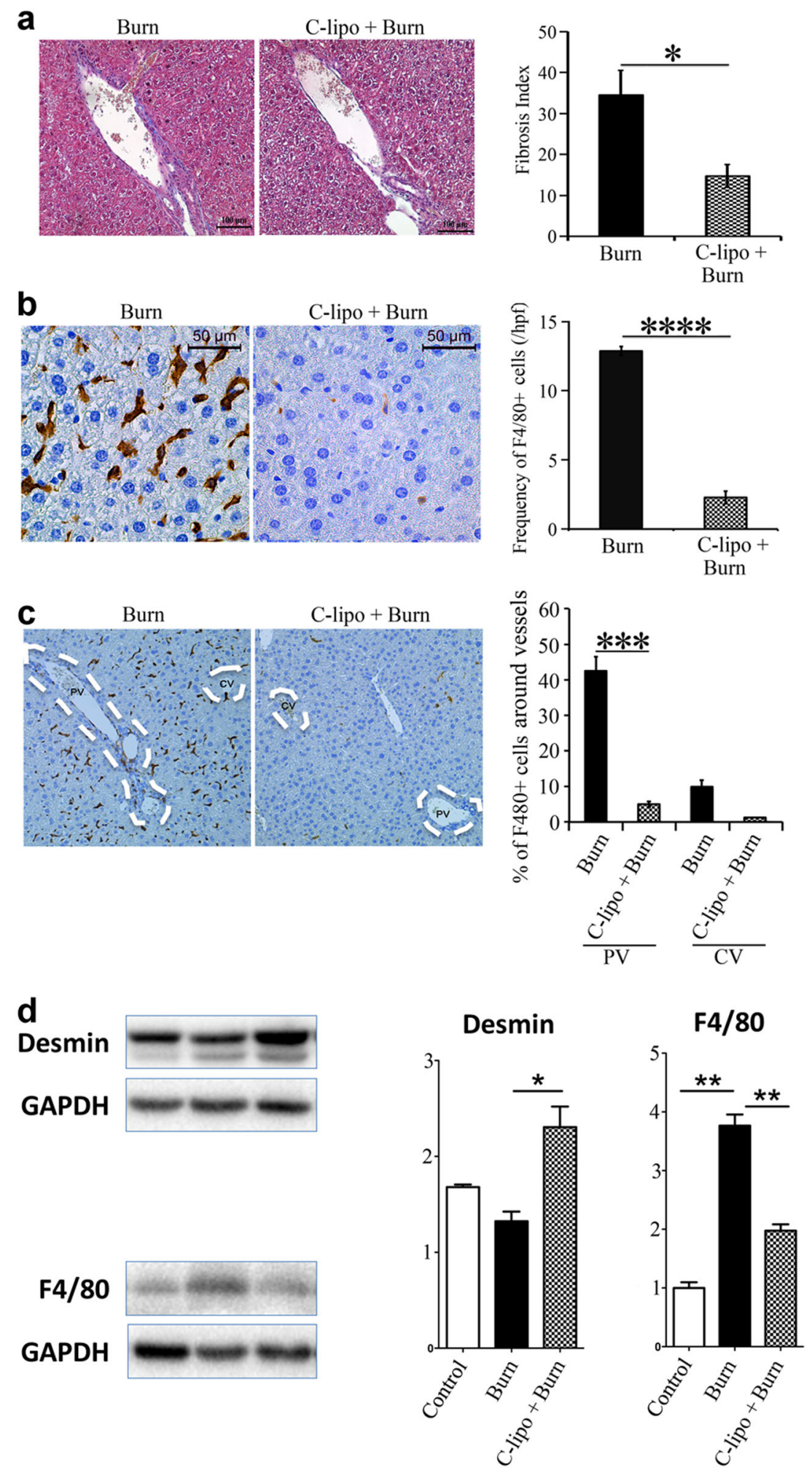

Fig. 3 Myeloid cells have an essential role in the induction of fibrosis post-thermal injury. a Mice treated with liposomal clodronate compared with control treated group. Trichrome staining shows less fibrosis around portal venules post-thermal injury when myeloid cells are depleted; $n=9$, scale bar $50 \mu \mathrm{m}$. b Immunohistochemistry shows a highly effective ablation of F4/80+ cells in liposomal clodronate-treated group in comparison to the control group; $n=9$, scale bar $50 \mu \mathrm{m}$. $\mathbf{c}$ There is a global ablation of F4/80+ cells in the liver of mice treated with liposomal clodronate, scale bar $100 \mu \mathrm{m}$. $\mathbf{d}$ In contrast to earlier findings, without F4/80+ cells, there is an increase in desmin + cells post-thermal injury; $n=3 .{ }^{*} P<0.05$, ${ }^{* *} P<0.01$, ${ }^{* * *} P<0.001,{ }^{* * * *} P<0.0001$ 

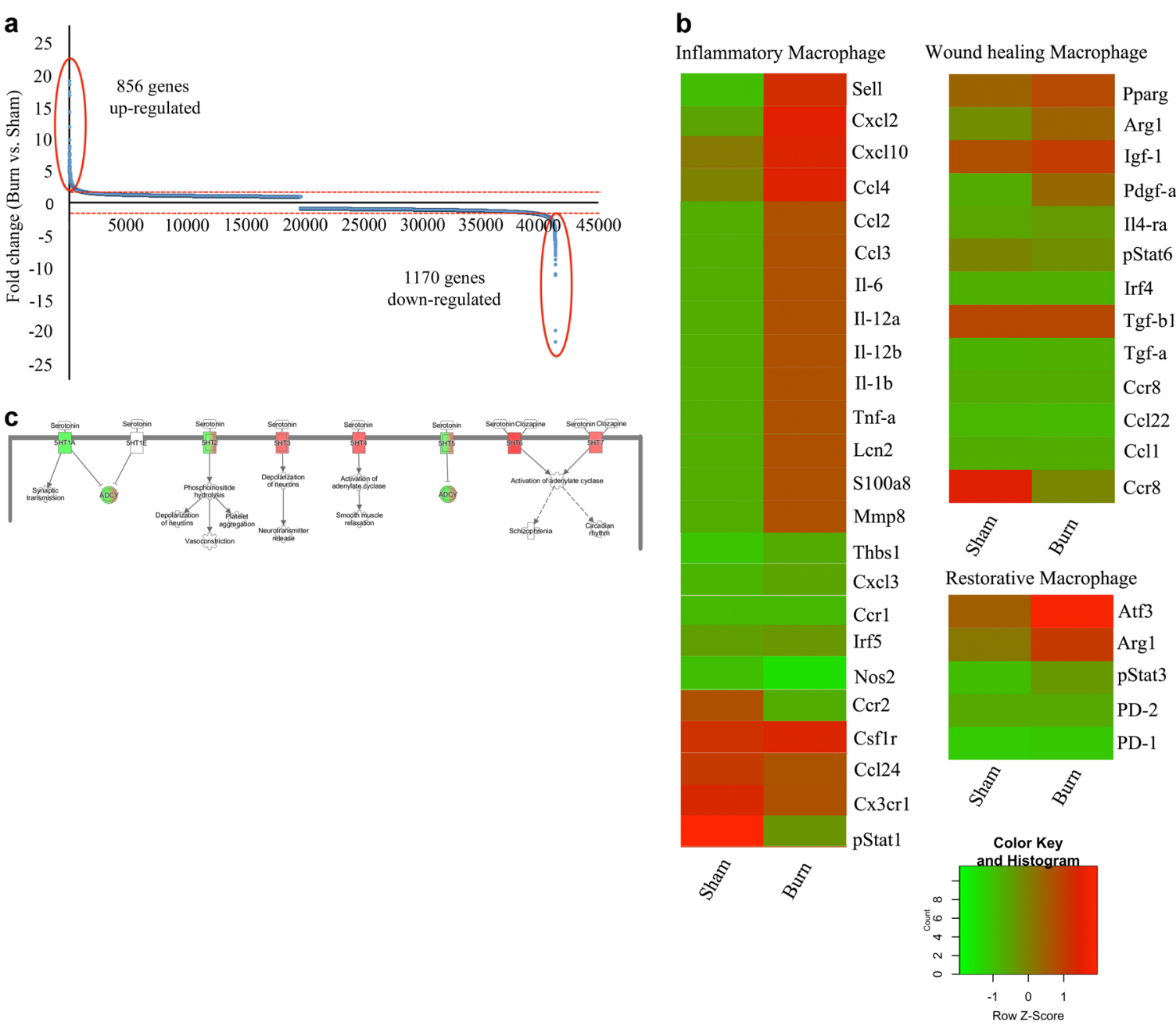

Fig. 4 EYFP + cells show a gene expression profile favoring a pro-inflammatory phenotype post-thermal injury. a There is a differential gene expression in $\sim 4.5 \%$ of genes when comparing burn and sham groups. $\mathbf{b}$ Pathway analysis shows upregulation of cytokines essential for stellate cell activation and (c) modulation of several components of the serotonin pathway, including the $5 \mathrm{HT}_{2 \mathrm{~A} / 2 \mathrm{C}}$ receptors

different zones of the liver using immunohistochemical analysis. The concentration of macrophages does not change after Ketanserin treatment (Fig. 5b). Moreover, the spatial pattern of these cells remained the same. Next, we asked if the phagocytic ability of macrophages is altered. We delivered fluorescently labeled zymosan particles to mature BMDM treated with and without Ketanserin. Although we did not observe a difference in the number of macrophages that were positive for zymosan (Fig. 5c), there was a reduction in the number of particles engulfed in macrophages treated with Ketanserin (Fig. 5d). Thus, we asked if there is an effect on the molecular signature of macrophages with Ketanserin treatment. Next, we treated BMDM with Ketanserin and looked at specific upregulated genes in the microarray in addition to key genes associated with M1 and M2 macrophages (Fig. 5e). To our surprise, in BMDM treated with Ketanserin there was a dramatic reduction in M2-associated gene IL-10 in addition to downregulation of macrophage mannose receptor (MMR) and TNF- $\alpha$ with up-regulation of M1 associated gene IL-6. Expression of pro-fibrotic factors TGF- $\beta 1$ and TGF- $\beta 3$ was not significantly different (Supporting Fig. 4C). LCN-2 showed a significant increase in BMDM treated with Ketanserin (Supporting Fig. 4C). However, we did not observe a significant difference between iNOS and Arg even though their ratio favors iNOS expression (Supporting Fig. 4C).

\section{Macrophages primed with ketanserin decrease pro-fibrotic characteritics of MSCs}

Knowing that activated resident MSCs of the liver, i.e., stellate cells, are the main producers of collagen and macrophages being the key regulators of their activation $^{13}$, we looked to see if BMDM primed with Ketanserin 


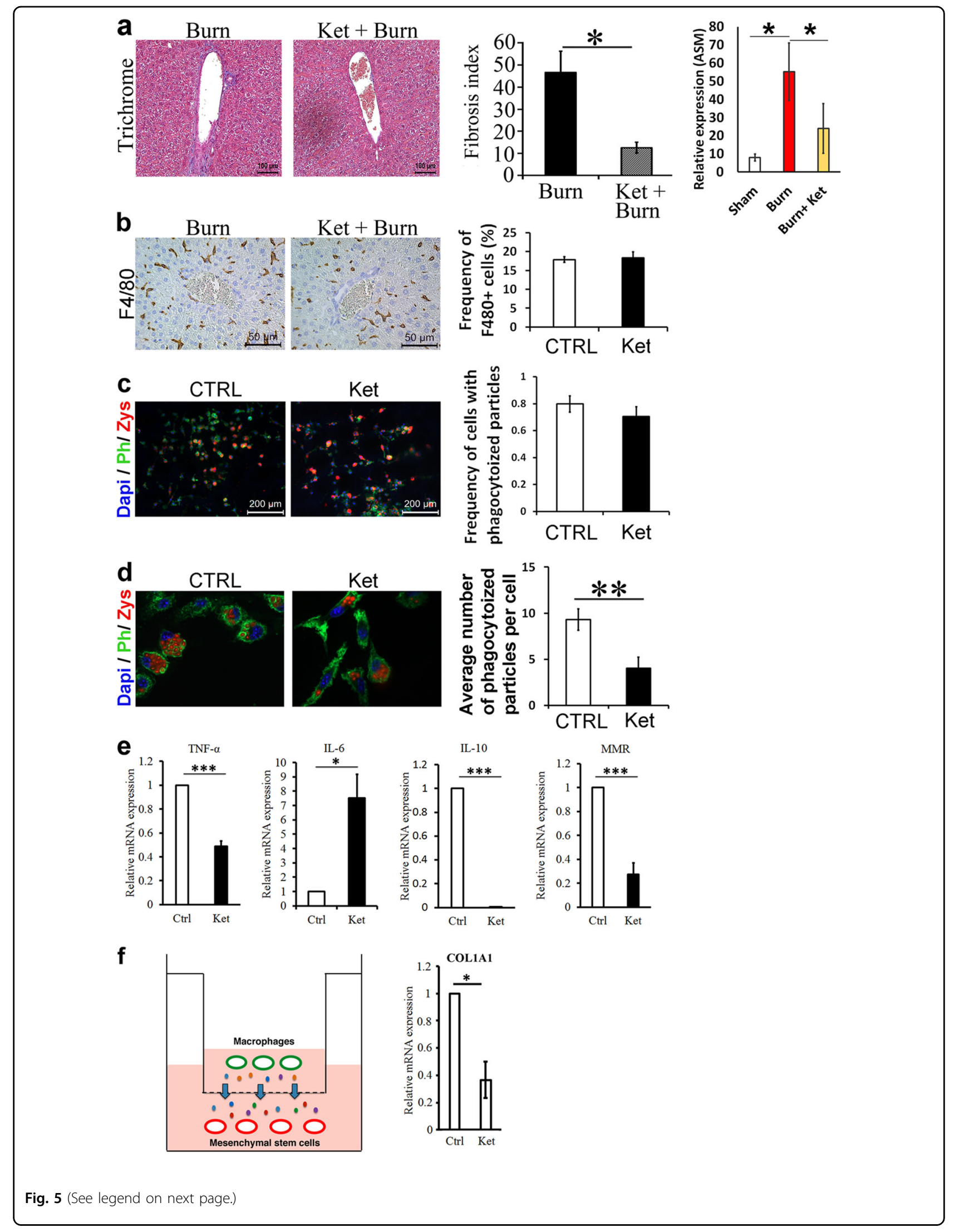


(see figure on previous page)

Fig. 5 Ketanserin decreases liver fibrosis post-thermal injury. a Trichrome staining shows less fibrosis post-thermal injury in mice treated with Ketanserin and qPCR analysis shows decreased expression of ASM; $n=5$, scale bar $100 \mu \mathrm{m} ; n=3$ per group for qPCR. $\mathbf{b}$ The accumulation and spatial pattern of macrophages do not change in the livers of mice treated with or without Ketanserin; $n=5$, scale bar $50 \mu \mathrm{m}$. $\mathbf{c}$ BMDM treated with and without ketanserin and then exposed to fluorescently labeled zymosan particles show no difference in the number of macrophages, which engulf zymosan particles; scale bar $200 \mu \mathrm{m}$. d BMDM treated with Ketanserin engulfed fewer zymosan particles compared to control. e qPCR analysis of relative mRNA expression of $\mathrm{M} 1$ and $\mathrm{M} 2$ associated genes of BMDM from healthy mice treated with vehicle or Ketanserin. Note that expression level in treated group (Ket) divided to control for each biological replicate. $\mathbf{f}$ Co-culture of mature BMDM, pretreated with vehicle or Ketanserin, with mature BMD-MSCs shows downregulation of Col1a1 expression in BMD-MSCs; scale bar $50 \mu \mathrm{m}$. ${ }^{*} P<0.05,{ }^{* *} P<0.01,{ }^{* * *} P<0.001$

would have less of a pro-fibrotic effect on bone-marrowderived MSCs (BMD-MSCs) of the same animal. After 3 days of co-culturing mature BMD-MSCs with mature BMDM pretreated with Ketanserin in vitro, MSCs had a decreased expression of mRNA levels of Colla1 compared to MSCs co-cultured with BMDM treated with vehicle as determined by qPCR analysis (Fig. $5 f$ ).

\section{Discussion}

A severe burn injury presents with numerous challenges. Burns greater than 30\% induce inflammation and hyper-metabolism, which carries on for 2 or more years after the injury, supposedly due to prolonged hepatic disturbances $^{25}$. What may be contributing to the prolonged hepatic disturbance? As organ fibrosis is generally a chronic condition, disturbances in the functional capacity of injured organs are extended for a longer duration ${ }^{26}$. Liver injury, such as after a severe burn, initiates a cascade of cellular and molecular responses, which may lead to tissue fibrosis and contribute to prolonged liver dysfunction. This could be a contributing factor to the prolonged hepatic disturbance observed post-thermal injury. To the best of our knowledge, this is the first paper that addresses this pathology in the liver after a severe thermal injury. Fibrosis becomes detrimental when there is prolonged damage resulting in excessive scarring and tissue dysfunction ${ }^{27,28}$. Whether fibrosis in the liver after burn is beneficial or detrimental is still not known and requires further investigation.

Here, we show that there is a mild spatiotemporal pattern of liver fibrosis post-thermal injury (Fig. 1a, b). We observed fibrosis predominately around portal venules, which peaked 7-14 days' post-thermal injury. Resolution of the excess ECM occurred by day 21 and remained low at day 42. Furthermore, there was an accumulation of EYFP+ myeloid cells in the liver postthermal injury, which suggests there is tissue injury (Fig. 2a, b). Myeloid cells accumulated in the liver, including around portal venules, where fibrosis was observed, highlighting a role for these cells during fibrosis post-thermal injury (Fig. 2c, d). Double staining for EYFP and BrdU suggests that the increase in myeloid cells is mainly due to recruitment rather than proliferation
(Supporting Fig. 1A-D), which is associated with driving the inflammatory response after tissue injury ${ }^{29}$. Moreover, the percentage of EYFP + cells in the bone marrow also increase after thermal injury (Supporting Fig. 2). When we ablated macrophages using liposomal clodronate there was less hepatic fibrosis and more quiescent stellate cells relative to when macrophages are present post-thermal injury (Fig. 3). This highlights the essential role of myeloid lineage cells during liver fibrosis. While recruitment and accumulation of myeloid cells are contributing in this fibrotic response, a phenotypic change in myeloid cells can also contribute to the pathology. Profiling of myeloid cells through microarray analysis suggests that these cells exist in a spectrum of states but more directed towards a pro-inflammatory phenotype in vivo (Fig. 4b). Whereas several signaling pathways have been altered in myeloid cells in the liver post-thermal injury, we observed a significant deregulation of several components of serotonin pathway, which suggested to us that targeting this pathway might change the phenotype of myeloid cells. Treating mice with Ketanserin led to significantly less fibrosis and down regulation of hepatic alpha-smooth muscle actin (ASM) expression (Fig. 5a) and no detrimental effect on liver function (Supporting Fig. 4B), suggesting Ketanserin can be a beneficiary prophylactic. The spatial pattern or migratory capacity of macrophages, unlike the observed fibrosis, does not seem to be impaired as the concentration of macrophages does not change after Ketanserin treatment (Fig. 5b). As such, the reduction of fibrosis may be due to the impaired phagocytic ability of macrophages treated with Ketanserin (Fig. 5c, d) as phagocytosis is essential for the conversion of inflammatory macrophages to their wound healing phenotype and subsequent upregulation of their pro-fibrotic secretome $\mathrm{e}^{30}$. This notion was supported by our in vitro experiment. At the mRNA level, we observed downregulation of TNF- $\alpha$, IL-10, MMR and upregulation of IL6 (Fig. 5e). Thus, Ketanserin appears to polarize BMDM more to a pro-inflammatory state despite their reduced phagocytic capacity. The final outcome of these changes is in the secretome, which induces less fibrosis in responsive cells (i.e., mesenchymal cells). This notion was further supported when we observed downregulation of the gene 
Col1a1 in BMD-MSCs co-cultured with mature BMDMs pretreated with Ketanserin in comparison to their control group in vitro (Fig. 5f). The cytokine profile of treated BMDM appears to have changed such that BMD-MSCs have a decreased capacity in expressing Col1a1, which is essential for collagen production. We decided to use BMD-MSCs because we can isolate them at a much higher concentration relative to HSCs, and recent evidence shows a high level of similarity between MSCs and $\mathrm{HSCs}^{31}$. Stellate cells can originate from the bone marrow, similar to MSCs. Furthermore, like MSCs in other organs, stellate cells are localized around endothelial cells. HSCs are being described as the resident MSCs of the liver due to comparable gene expression profiles and inherent capacity to differentiate into adipocytes or osteocytes, and properties that promote extra-medullary hematopoiesis $^{32,33}$. LCN-2 is a known marker for macrophage deactivation ${ }^{34}$ and was upregulated in macrophages conditioned with Ketanserin (Supporting Fig. 4C), which may be having a role in altering the functional capacity of macrophages in driving fibrosis. A burn injury has a profound systemic effect at different cellular and hormonal levels ${ }^{35-40}$. In vitro, we observe a similar response in burn-derived macrophages treated with Ketanserin relative to their sham counterparts (Supporting Fig. 4D). Thus, even in burn-derived macrophages, Ketanserin is skewing the phenotype of these cells away from their M2 "wound-healing" phenotype. However, more research is warranted on the effects of Ketanserin in vivo, As our study does not demonstrate a direct effect of macrophages on desmin-expressing cells in vivo.

Studies suggest that 5-HT has good and bad implications for the liver ${ }^{41}$. Treating rodents with $5 \mathrm{HT}$ after a $2 / 3$ partial hepatectomy accelerated liver regeneration ${ }^{18}$ and boosted tissue repair after ischemia/reperfusion injury ${ }^{42}$. In humans undergoing liver resection, intra-platelet $5 \mathrm{HT}$ is shown to be associated with enhanced liver regeneration and maybe a good clinical indicator of post-operative liver dysfunction ${ }^{43}$. Furthermore, $5 \mathrm{HT}$ protects against small-for-size liver graft failure by maintaining hepatic circulation allowing for enhanced liver regeneration ${ }^{44}$. In aged mice, a 5HT receptor agonist rescues deficient liver regeneration ${ }^{45}$. Despite these promising findings, $5 \mathrm{HT}$ has also been shown to be a critical pro-fibrotic factor through the promotion of HSC proliferation ${ }^{18}$. Ketanserin, a $5 \mathrm{HT}_{2 \mathrm{~A} / 2 \mathrm{C}}$ receptor antagonist has been shown to impair HSC activation and reduce liver fibrosis ${ }^{46}$. However, its effect in vivo on macrophages, the regulators of HSC activation and fibrosis, is not known.

Beside a deregulation in components of serotonin pathway, our microarray analysis on isolated EYFP+ myeloid cells identified several altered signaling pathways in the livers of injured animals (Supporting Fig. 5). Farnesoid $\mathrm{x}$ receptor (FXR) and retinoid $\mathrm{x}$ receptor (RXR) pathways were three of the top five deregulated canonical pathways in myeloid cells isolated from the liver of injured animals. The farnesoid X receptor (FXR) is highly expressed in the liver and intestine. FXR has an essential role in gut-liver axis feedbacks regulating lipid and glucose homeostasis ${ }^{47}$. In addition, it is shown to be a powerful regulator of several hepatic metabolic pathways relevant to fatty liver disease and cholestasis ${ }^{48,49}$. A consequence of cholestasis is the accumulation of bile acids, which promotes inflammation and a pro-fibrogenic response. Furthermore, the forth-highest activated pathway was the coagulation system, which along with the accumulation of bile acids may explain why there is fibrosis in the liver post-thermal injury. Overall, the observed pathways suggest that myeloid cells have a diverse role in liver pathology post-thermal injury.

An increase in monocyte-derived hepatic macrophages in response to tissue injury has been well documented ${ }^{50-52}$. Studies suggest that pro-fibrogenic macrophages are derived from this population. These macrophages produce

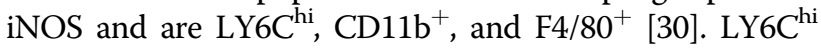
monocyte-derived macrophages have been shown to be the main pro-fibrotic macrophages in numerous tissues including the liver ${ }^{53-55}$. Our data support the notion that accumulated myeloid cells in the liver are due to recruitment of these cells, which is associated with liver fibrosis. Furthermore, inhibiting the $5 \mathrm{HT}_{2 \mathrm{C}}$ receptor impairs mRNA expression of $\mathrm{CCL} 2^{56}$, a potent cytokine for monocyte recruitment ${ }^{57}$. Ketanserin is a known $5 \mathrm{HT}_{2 \mathrm{~A} / 2 \mathrm{C}}$ receptor antagonist, which may consequently be impairing recruitment of pro-fibrotic LY6C ${ }^{\text {hi }}$ monocytes to the liver, suggesting another possible mechanism for the anti-fibrotic effect of Ketanserin in the liver.

In conclusion, we describe novel phenomena after a severe thermal injury in the liver. Whether this fibrotic response is protective or pathologic is yet to be determined. Nevertheless, we show an essential role in myeloid lineage cells mapping out fibrosis in the liver. 5HT antagonist Ketanserin appears to change the molecular signature of macrophages in a way that impairs this profibrotic response. While we observe a beneficial effect of Ketanserin in inhibiting liver fibrosis, it would be essential to unravel its role on other organs, particularly in the skin, which is the first damaged organ in burn patients. However, we did not observe any change in AST/ALT, animal weight, nor general wellness of the animals between control and treated groups. If detrimental effects are observed in other organs with Ketanserin treatment, then tissue-specific delivery of Ketanserin would a safer treatment approach.

\footnotetext{
Acknowledgements

This study was supported by Canadian Institutes of Health Research \# 123336. CFI Leader's Opportunity Fund: Project \# 25407 NIH RO1 GM087285-01. The
} authors would like to thank Dr. Benjamin Alman for allowing us to use the 
myeloid lineage reporter mice for this study. Address for Reprints: Same as corresponding author, please see above. Conflicts of Interest and Source of Funding: This study was supported by -Canadian Institutes of Health Research \# 123336. NIH RO1 GM087285-01, Toronto Hydro donation, EMH SEED grant.

\section{Author details}

'Sunnybrook Research Institute, Toronto, Canada. ${ }^{2}$ Laboratory Medicine and Pathobiology, University of Toronto, Toronto, Canada. ${ }^{3}$ Department of Surgery, Division of Plastic Surgery, University of Toronto, Toronto, Canada. ${ }^{4}$ Institute of Medical Science, University of Toronto, Toronto, Canada. ${ }^{5}$ Department of Immunology, University of Toronto, Toronto, Canada. ${ }^{6}$ Ross-Tilley Burn Centre, Sunnybrook Health Sciences Centre, Toronto, Canada

\section{Author contributions}

S.A.N., A.R.S., and M.G.J. were involved in designing the study and interpreting the data. Experiments were performed by A.R.S., S.A.N., L.D., and C.B. A.R.S. and S.A.N. wrote the manuscript. M.G.J. obtained funding and performed a critical revision of the manuscript.

\section{Conflict of interest}

The authors declare that they have no conflict of interest.

\section{Publisher's note}

Springer Nature remains neutral with regard to jurisdictional claims in published maps and institutional affiliations.

Supplementary information accompanies this paper at https://doi.org/ 10.1038/s12276-018-0118-x.

Received: 10 November 2017 Revised: 3 April 2018 Accepted: 9 April 2018 Published online: 19 July 2018

\section{References}

1. Jeschke, M. G. The hepatic response to thermal injury: is the liver important for postburn outcomes? Mol. Med. 15, 337-351 (2009).

2. Gauglitz, G. G. et al. Post-burn hepatic insulin resistance is associated with endoplasmic reticulum (ER) stress. Shock 33, 299-305 (2010).

3. Jeschke, M. G. et al. Severe injury is associated with insulin resistance, endoplasmic reticulum stress response, and unfolded protein response. Ann. Surg. 255, 370-378 (2012).

4. Pellicoro, A., Ramachandran, P., Iredale, J. P. \& Fallowfield, J. A. Liver fibrosis and repair: immune regulation of wound healing in a solid organ. Nat. Rev. Immunol. 14, 181-194 (2014).

5. Das, A. et al. Monocyte and macrophage plasticity in tissue repair and regeneration. Am. J. Pathol. 185, 2596-2606 (2015).

6. Bourbonnais, E. et al. Liver fibrosis protects mice from acute hepatocellular injury. Gastroenterology 142, 130-139 e134 (2012).

7. Amini-Nik, S. et al. beta-Catenin-regulated myeloid cell adhesion and migration determine wound healing. J. Clin. Invest. 124, 2599-2610 (2014).

8. Duffield, J. S. et al. Selective depletion of macrophages reveals distinct, opposing roles during liver injury and repair. J. Clin. Invest. 115, 56-65 (2005).

9. Lavine, K. J. et al. Distinct macrophage lineages contribute to disparate patterns of cardiac recovery and remodeling in the neonatal and adult heart. Proc. Natl Acad. Sci. USA 111, 16029-16034 (2014).

10. Gordon, S. \& Taylor, P. R. Monocyte and macrophage heterogeneity. Nat. Rev. Immunol. 5, 953-964 (2005).

11. Tacke, F. \& Zimmermann, H. W. Macrophage heterogeneity in liver injury and fibrosis. J. Hepatol. 60, 1090-1096 (2014).

12. Mosser, D. M. \& Edwards, J. P. Exploring the full spectrum of macrophage activation. Nat. Rev. Immunol. 8, 958-969 (2008).

13. Wynn, T. A. \& Barron, L. Macrophages: master regulators of inflammation and fibrosis. Semin. Liver. Dis. 30, 245-257 (2010).

14. Mossner, R. \& Lesch, K. P. Role of serotonin in the immune system and in neuroimmune interactions. Brain Behav. Immun. 12, 249-271 (1998).

15. Lesurtel, M., Soll, C., Graf, R. \& Clavien, P. A. Role of serotonin in the hepatogastrolntestinal tract: an old molecule for new perspectives. Cell. Mol. Life Sci. 65, 940-952 (2008).
16. Ebrahimkhani, M. R. et al. Stimulating healthy tissue regeneration by targeting the 5-HT(2)B receptor in chronic liver disease. Nat. Med. 17, 1668-1673 (2011).

17. Lesurtel, M. et al. Platelet-derived serotonin mediates liver regeneration. Science 312, 104-107 (2006).

18. Ruddell, R. G. et al. A role for serotonin (5-HT) in hepatic stellate cell function and liver fibrosis. Am. J. Pathol. 169, 861-876 (2006).

19. Sadiq, A. et al. The role of serotonin during skin healing in post-thermal injury. Int. J. Mol. Sci. 19, 1034 (2018).

20. Abdullahi, A., Amini-Nik, S. \& Jeschke, M. G. Animal models in burn research. Cell Mol. Life Sci. 71, 3241-3255 (2014).

21. Amini-Nik, S. et al. Pax7 expressing cells contribute to dermal wound repair, regulating scar size through a beta-catenin mediated process. Stem Cells $\mathbf{2 9}$, 1371-1379 (2011).

22. Arno, A. I. et al. Human Wharton's jelly mesenchymal stem cells promote skin wound healing through paracrine signaling. Stem Cell Res. Ther. 5, 28 (2014).

23. Van Rooijen, N. \& Sanders, A. Liposome mediated depletion of macrophages: mechanism of action, preparation of liposomes and applications. J. Immunol. Methods 174, 83-93 (1994).

24. Bedossa, P. \& Poynard, T. An algorithm for the grading of activity in chronic hepatitis C. The METAVIR Cooperative Study Group. Hepatology 24, 289-293 (1996).

25. Jeschke, M. G. et al. Long-term persistance of the pathophysiologic response to severe burn injury. PLOS ONE 6, e21245 (2011).

26. Rockey, D. C., Bell, P. D. \& Hill, J. A. Fibrosis-a common pathway to organ injury and failure. N. Engl. J. Med. 373, 96 (2015).

27. Amini-Nik, S., Yousuf, Y. \& Jeschke, M. G. Scar management in burn injuries using drug delivery and molecular signaling: current treatments and future directions. Adv. Drug Deliv. Rev. 123, 135-154 (2018).

28. Bielefeld, K. A., Amini-Nik, S. \& Alman, B. A. Cutaneous wound healing: recruiting developmental pathways for regeneration. Cell Mol. Life Sci. 70 2059-2081 (2013).

29. Epelman, S. et al. Embryonic and adult-derived resident cardiac macrophages are maintained through distinct mechanisms at steady state and during inflammation. Immunity 40, 91-104 (2014).

30. Nacu, N. et al. Macrophages produce TGF-beta-induced (beta-ig-h3) following ingestion of apoptotic cells and regulate MMP14 levels and collagen turnover in fibroblasts. J. Immunol. 180, 5036-5044 (2008).

31. Kordes, C., Sawitza, I., Gotze, S., Herebian, D. \& Haussinger, D. Hepatic stellate cells contribute to progenitor cells and liver regeneration. J. Clin. Invest. 124, 5503-5515 (2014)

32. Kordes, C., Sawitza, I., Gotze, S. \& Haussinger, D. Hepatic stellate cells support hematopoiesis and are liver-resident mesenchymal stem cells. Cell. Physiol. Biochem. 31, 290-304 (2013)

33. Castilho-Fernandes, A. et al. Human hepatic stellate cell line (LX-2) exhibits characteristics of bone marrow-derived mesenchymal stem cells. Exp. Mol. Pathol. 91, 664-672 (2011).

34. Warszawska, J. M. et al. Lipocalin 2 deactivates macrophages and worsens pneumococcal pneumonia outcomes. J. Clin. Invest. 123, 3363-3372 (2013).

35. Jeschke, M. G. et al. Pathophysiologic response to severe burn injury. Ann. Surg. 248, 387-401 (2008).

36. Patsouris, D. et al. Burn Induces Browning of the Subcutaneous White Adipose Tissue in Mice and Humans. Cell Rep. 13, 1538-1544 (2015).

37. Stanojcic, M. et al. Leukocyte infiltration and activation of the NLRP3 inflammasome in white adipose tissue following thermal injury. Crit. Care Med. $\mathbf{4 2}$ 1357-1364 (2014)

38. Stanojcic, M., Chen, P., Xiu, F. \& Jeschke, M. G. Impaired immune response in elderly burn patients: new insights into the immune-senescence phenotype. Ann. Surg. 264, 195-202 (2016)

39. Abdullahi, A. et al. IL-6 Signal from the bone marrow is required for the browning of white adipose tissue post burn injury. Shock 47, 33-39 (2017).

40. Diao, L. et al. Alternative mechanism for white adipose tissue lipolysis after thermal injury. Mol. Med. https://doi.org/10.2119/molmed.2015.00123 (2015) .

41. Lesurtel, M., Soll, C., Humar, B. \& Clavien, P. A. Serotonin: a double-edged sword for the liver? Surgeon 10, 107-113 (2012).

42. Nocito, A. et al. Platelets and platelet-derived serotonin promote tissue repair after normothermic hepatic ischemia in mice. Hepatology 45, 369-376 (2007).

43. Starlinger, P. et al. Evidence for serotonin as a relevant inducer of liver regeneration after liver resection in humans. Hepatology 60, 257-266 (2014).

44. Tian, $Y$. et al. Activation of serotonin receptor-2B rescues small-for-size liver graft failure in mice. Hepatology 53, 253-262 (2011). 
45. Furrer, $K$. et al. Serotonin reverts age-related capillarization and failure of regeneration in the liver through a VEGF-dependent pathway. Proc. Natl Acad. Sci. USA 108, 2945-2950 (2011).

46. Kim, D. C. et al. 5-HT2A receptor antagonists inhibit hepatic stellate cell activation and facilitate apoptosis. Liver. Int. 33, 535-543 (2013).

47. Jiang, $C$. et al. Intestinal farnesoid $X$ receptor signaling promotes nonalcoholic fatty liver disease. J. Clin. Invest. 125, 386-402 (2015).

48. Modica, S., Gadaleta, R. M. \& Moschetta, A. Deciphering the nuclear bile acid receptor FXR paradigm. Nud. Recept. Signal. 8, e005 (2010).

49. Sinal, C. J. et al. Targeted disruption of the nuclear receptor FXR/BAR impairs bile acid and lipid homeostasis. Cell 102, 731-744 (2000).

50. Dal-Secco, D. et al. A dynamic spectrum of monocytes arising from the in situ reprogramming of CCR2+monocytes at a site of sterile injury. J. Exp. Med. 212, 447-456 (2015).

51. Karlmark, K. R. et al. Hepatic recruitment of the inflammatory Gr1 + monocyte subset upon liver injury promotes hepatic fibrosis. Hepatology 50, 261-274 (2009).
52. Heymann, F. et al. Hepatic macrophage migration and differentiation critical for liver fibrosis is mediated by the chemokine receptor $\mathrm{C}-\mathrm{C}$ motif chemokine receptor 8 in mice. Hepatology 55, 898-909 (2012).

53. Gibbons, M. A. et al. Ly6Chi monocytes direct alternatively activated profibrotic macrophage regulation of lung fibrosis. Am. J. Respir. Crit. Care. Med. 184 569-581 (2011)

54. Ramachandran, P. et al. Differential Ly-6C expression identifies the recruited macrophage phenotype, which orchestrates the regression of murine liver fibrosis. Proc. Natl Acad. Sci. USA 109, E3186-E3195 (2012).

55. Imamura, M., Ogawa, T., Sasaguri, Y., Chayama, K. \& Ueno, H. Suppression of macrophage infiltration inhibits activation of hepatic stellate cells and liver fibrogenesis in rats. Gastroenterology 128, 138-146 (2005).

56. Mikulski, Z. et al. Serotonin activates murine alveolar macrophages through 5-HT2C receptors. Am. J. Physiol. Lung Cell Mol. Physiol. 299, L272-L280 (2010).

57. Qian, B. Z. et al. CCL2 recruits inflammatory monocytes to facilitate breasttumour metastasis. Nature 475, 222-225 (2011). 